\title{
Suspended Cell Patterning for Automatic Microrobotic Cell Injection
}

\author{
W.H. Wang, Member, IEEE, M.M. Alkaisi, J.G. Chase, X.Q. Chen, Senior Member, IEEE, C.E. Hann
}

\begin{abstract}
Microinjection of DNA/mRNA/morpholinos is a critical technology for molecular biology and drug discovery. When dealing with suspended cells, state-of-the-art manual injection involves a time-consuming and tedious sample preparation procedure, to accurately align cells. To enable automatic microrobotic cell injection, this paper reports on two inexpensive, reusable, biocompatible, and easy-to-make devices that are capable of patterning a large number of cells in 10-30 seconds. One device is based on negative air pressure and made of polycarbonate using a conventional micro-machining process. It is particularly suitable for cells larger than $100 \mu \mathrm{m}$, such as the zebrafish embryo patterning and successful gene 'knock-down' products of the morpholino-injected embryos. The other device is based on dielectrophoresis and suitable for cells smaller than $100 \mu \mathrm{m}$, demonstrated by successful trapping of pituitary cells. These devices offer a complete solution for suspended cells in all size spectrums to be prepared up to 10 times faster than manual human preparation. Furthermore, this approach can facilitate high-throughput automatic microrobotic cell injection, for injection applications such as the injection of zebrafish embryos, mouse oocytes/embryos, Drosophila embryos, and other types of suspended cells.
\end{abstract}

Index Terms - Cell injection, cell patterning, fluidic device, dielectrophoresis, microrobotics.

\section{INTRODUCTION}

Molecule screening at the single cell level is critical in molecular biology and drug discovery. It requires that target molecules be introduced into single cells to permit cellular-function-targeted molecules to directly regulate cell development and their functions to be quantified. Several technologies exist for introducing foreign materials into a cell, such as electroporation [1], viral vectors [2], gene gun [3], ultrasonics [4], and MEMS-based injection [5], [6]. Compared to these techniques, manual microinjection with a single glass micropipette remains the most effective in terms of cell damage, cell viability, cell waste, effectiveness of delivering macromolecules, specificity, and freedom from concerns about phenotype alteration.

In the laboratory, manual injection is the state-of-the-art technique for DNA/mRNA/MO injection. There are problems inherent in manual injection, such as human fatigue and large variations in success rates due to poor reproducibility. To enable fast, precise, and robust screening for molecular targets, a fully automated microrobotic cell injection system has been developed [7].

Wang, Chase, Chen, and Hann are with the Dept. of Mechanical Engineering, University of Canterbury, Private Bag 4800, Christchurch 8140, New Zealand (e-mail: wenhui.wang@canterbury.ac.nz).

Alkaisi is with the Dept. of Electrical and Computer Engineering, University of Canterbury, Private Bag 4800, Christchurch 8140, New Zealand. (e-mail: m.alkaisi@elec.canterbury.ac.nz).



Fig. 1. Suspended cell arranged in a regular pattern for automatic microrobotic cell injection. Cells are patterned into gridded locations by trapping force.

Cells can be broadly classified as suspended cells (e.g., embryos/oocytes, red blood cells) and adherent cells (e.g., HeLa cells, fibroblasts, and endothelial cells). Adherent cells adhere to the bottom surface of a culture dish/plate during in vitro culture, not requiring immobilization efforts. However, their irregular morphology makes robust pattern recognition more difficult and full automation is therefore much more challenging. As the first step, suspended cells are chosen for the development of the automatic cell injection system.

The current practice of preparing the cells in state-ofthe-art manual injection systems is to manually line cells into rows (e.g., in preparing zebrafish embryos, a technician carefully tweaks them into agarose trough [8]), which is time-consuming ( $\sim 2$ minutes for 30 cells) and tedious. Therefore, to automate cell injection, the first effort should be made to immobilize the suspended cells into a regular pattern (Fig. 1), which will significantly simplify the microrobotbased embryo searching/positioning task and thus increase the injection speed. In this paper, without loss of generality, the cells are patterned in gridded locations. However, they could be readily adapted to specific applications as required. The cells can be either immobilized on through-holes or trapped on cavities.

Suspended cells have dimensions ranging from $5 \mu \mathrm{m}$ to $\sim 1 \mathrm{~mm}$. There does not exist a universal method/device to pattern cells in various dimensions. For cells smaller than 100 $\mu \mathrm{m}$, dielectrophoresis (DEP) trapping [9] and laser trapping [10] are suitable, as the force generated by the electrical field or laser is strong enough to manipulate cells. For cells larger than $100 \mu \mathrm{m}$, these methods require higher power to generate sufficient manipulation force. Therefore, they complicate the device construction and more importantly, may produce undesired biological complications for further cellular development. It is thus highly desirable to design 
and fabricate two different, low cost devices to provide a complete solution to patterning cells of all sizes.

In this paper, zebrafish embryos are used for large cells, and a pressure-based fluidic device is introduced to pattern the embryonic cells. The zebrafish has emerged as an important model organism for developmental genetic studies as well as for drug discovery [11]. The embryos are transparent, making it convenient to observe the movement and fate of individual cells during embryonic development [12]. Molecular and genetic analyses of zebrafish embryogenesis depend on the injection of foreign materials into early zebrafish embryos [13], such as DNA, mRNA, morpholinomodified oligonucleotides (morpholinos or MOs), for in vivo [14] study. To verify the device, a number of embryos are immobilized by the device and then injected by the automatic cell injection system [7].

To extend the automatic cell injection system to small cells, a dielectrophoresis-based MEMS biochip has been developed. A pressure-based mechanism is not used for two reasons: i) Because the cell is small, the size of throughholes for providing the sucking force should be very small (e.g., $3 \mu \mathrm{m}$ for cells in a diameter of $15 \mu \mathrm{m}$ ). Fabrication (e.g., by laser ablation [15]) of these small through-holes is highly cost and time expensive. ii) In line with the small size of cells, the thickness of device will be approximately $100 \mu \mathrm{m}$. The deformation of the device surface caused by the required pressure forces will affect cell visibility for automatic injection. The biochip developed has been proven to be able to immobilize individual cells into cavities, laying the foundation for future work.

In this paper, the microrobotic cell injection system is briefly introduced first. Then, the pressure-based fluidic device for immobilizing big cells is discussed with experimental results of cell patterning and injection. Next, the dielectrophoresis-based MEMS cell patterning biochip is presented with cell trapping results. The paper ends with a summary conclusion.

\section{MiCROROBOTIC CELL INJECTION S YSTEM}

\section{A. System Structure}

Fig. 2 shows the general structure for a suspended cell injection system. Two motorized positioners 1, 2, such as multi-DOF motorized positioning stages or microrobots/micromanipulators, control the motion of cells and micropipette respectively. A control software unit runs on the host computer for motion control and image processing. Position control devices connected to, or mounted on, the host computer physically provide control signals to the two positioners and the pressure unit. A cell patterning device is placed on positioner 1 , and an injection micropipette (glass capillary or microfabricated needle) is attached to the second positioner 2. A CCD/CMOS camera is mounted on the optical microscope to obtain the cell images. The computer-controlled pressure unit is responsible to apply pressure pulses to the micropipette for material injection. All the units except the host computer and pressure unit are mounted on a anti-vibration table to minimize vibration.

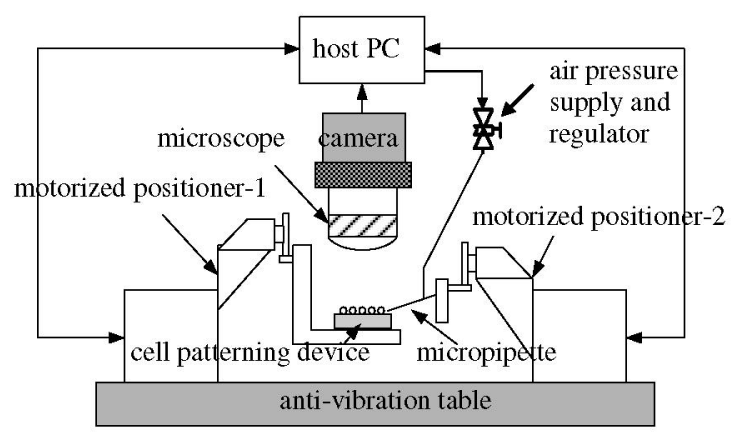

Fig, 2. The general structure of a microrobotic cell injection system.

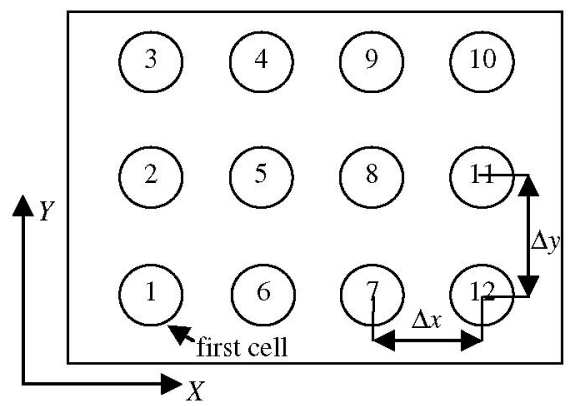

Fig. 3. Injection paths. Path-1: 1-2-3-4-5-6-7-8-9-10-11-12 with distance $d_{1}$. Path-2: 1-6-7-12-11-8-5-2-3-4-9-10 with distance $d_{2}$. Path-3: 1-2-3-4-910-11-12-7-6-5-8 with distance $d_{3}$. Path-4: 1-6-7-12-11-10-9-4-3-2-5-8 with distance $d_{4}$.

Before injection is started, all cells are immobilized on the cell patterning device to facilitate the task of searching and positioning cells, which is performed by the positioner. Using an appropriate magnification (dependent on the cell size: e.g., $2.4 \times$ for zebrafish embryos, and $40 \times$ for pituitary cells), only one cell appears in the field of view of the camera, and its image is processed for structure recognition, which provides the application-determined injection destinations.

\section{B. Injection Path Selection}

Upon the patterning of cells in a gridded manner, the system sequentially conducts injection. As illustrated in Fig. 3, a microrobot (Sutter MP-285, CA, USA) can be controlled to travel along certain paths, out of which the shortest path should be selected to minimize injection time per cell. The four labeled paths in Fig. 3 represent the most efficient candidates for sequential batch injection, and are considered here. Denote by $\Delta x$ and $\Delta y$ the pitch between adjacent cells along the $X$ and $Y$ directions, and denote by $m$ and $n$ the number of cells along the $X$ and $Y$ directions. The lengths of the four paths are thus:

$$
\begin{gathered}
d_{1}=m(n-1) \Delta y+(m-1) \Delta x \\
d_{2}=n(m-1) \Delta x+(n-1) \Delta y \\
d_{3}= \begin{cases}\left(n-1+C_{n}^{2}-C_{n-m+1}^{2}\right) \Delta y+C_{m}^{2} \Delta x & \text { if } n \geq m \\
\left(n-1+C_{n}^{2}\right) \Delta y+\left(C_{m}^{2}-C_{m-n}^{2}\right) \Delta x & \text { else }\end{cases} \\
d_{4}= \begin{cases}\left(m-1+C_{m}^{2}\right) \Delta x+\left(C_{n}^{2}-C_{n-m}^{2}\right) \Delta y & \text { if } n \geq m \\
\left(m-1+C_{m}^{2}-C_{m-n+1}^{2}\right) \Delta x+C_{n}^{2} \Delta y & \text { else }\end{cases}
\end{gathered}
$$



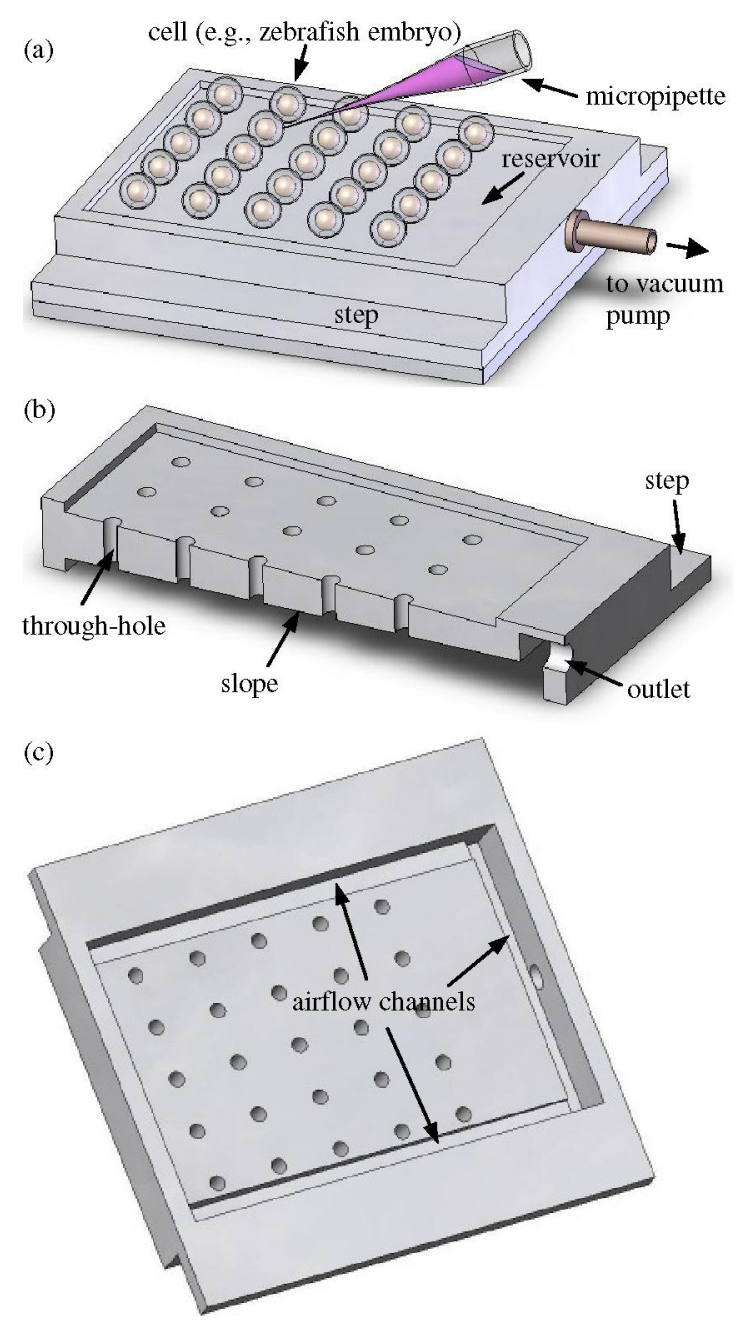

Fig. 4. A pressure-based fluidic device for patterning biological cells. (a) Scheme of the immobilized cells on the device for micropipette injection. (b) Section view of the cell sucking structure. (c) Bottom 3-D view of the cell sucking structure.

where $C_{x}^{y}$ is the binomial coefficient.

If a cell patterning device is designed to provide $\Delta y<\Delta x$, $m>2$, and $n>2$, it will result in $d_{1}<d_{3}<d_{4}<d_{2}$. As path- 1 is the shortest, it is selected as the injection sequence.

\section{PRESSURE-BASED FLuidic DEVICE For BIG EMBRYONIC CELLS}

\section{A. Device Design}

Fig. 4 shows one example vacuum-based device, which has two parts: cell sucking structure, and a flat piece glued on the bottom. Arrays of through holes are used to immobilize zebrafish embryos with negative pressure applied through the air outlet on the side wall of the enclosed chamber. For zebrafish embryos, which are $\sim 1.2 \mathrm{~mm}$ in diameter including chorion, the diameter of the through holes is $400 \mu \mathrm{m}$.

A reservoir is designed to contain culture media/solution throughout the injection process. A slope on the bottom surface of the holes is created for air bubbles to escape more readily so they do not stick to the bottom surface.

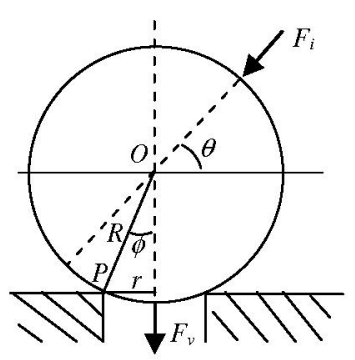

Fig. 5. Schematic for negative pressure estimation.

Air bubbles beneath the through-holes lead to poor image qualities, which make the image processing for cellular structure recognition unnecessarily complex or impossible. The three airflow channels along the bottom surface are for inducing air to smoothly flow out of the chamber via the air outlet. The air outlet is positioned higher than the slope to guarantee that the slope is submerged in culture media/solution. This configuration aims to obtain uniform image intensity and minimize lighting distortion for all cells by allowing for the illumination to go through the same media. The steps are created such that the cell patterning device can be fixed by two clamps under the microscope.

Polycarbonate is chosen for constructing the cell patterning device because it is optically transparent, biocompatible, inexpensive, and easily machined. Apart from these advantages, the toughness of polycarbonate ensures that the device is strong enough to withstand the applied negative pressure without significant deformation.

\section{B. Sucking Pressure Estimation}

As shown in Fig. 5, the pressure required to stabilize the cell, which is assumed as a rigid object, can be obtained when equilibrium is maintained during injection. Assume the penetration is always through the cell center with a titling angle with respect to the horizontal plane, let $F_{i}$ be the penetration force, $F_{v}$ the sucking force, $O$ the center of the cell, $P$ the supporting point, $R$ the radius of the cell, and $r$ the radius of the through-hole. The nominal negative pressure is thus:

$$
P_{v}=R \cos (\theta+\phi) F_{i} /\left(\pi r^{3}\right)
$$

where $\phi=\sin ^{-1}(r / R)$.

For a typical tilting angle $\theta=45^{\circ}$, and the zebrafish embryo case parameters, substituting $R=600 \mu \mathrm{m}, r=200 \mu \mathrm{m}$, $F_{i}=700 \mu \mathrm{N}[16]$ yields a required nominal pressure of 54.6 $\mathrm{mmHg}$.

Preferably, the negative pressure applied to immobilizing embryos should be low enough not to cause damage or negative effects for embryonic development. To determine an appropriate level of pressure, 200 embryos were tested. It was found that for $\theta=45^{\circ}$, a negative pressure of 12.7-25.4 mmHg proved effective in immobilizing zebrafish embryos of different strains without damaging the embryos. In an extreme experiment, embryos were deliberately applied with the negative pressure of $25.4 \mathrm{mmHg}$ continuously for 30 minutes. Although the chorion was indeed subject to shape 




Fig. 6. The integral pressure-based fluidic cell patterning device.

distortion, the embryos developed to normal fish as those control embryos did. Nevertheless, in practice embryos will never be exposed to the pressure for 30 minutes. Instead, for example, injection of 100 embryos patterned by such a device with the developed microrobotic injection system, which is capable of injecting embryos at a speed of 15 embryos/minute, takes about 7 minutes. It is therefore safe to use this level of sucking pressure for patterning cells without worrying about adverse effects.

\section{Micromachining of the Device}

On one side of the device opposite to the outlet is embedded a glass substrate of $0.5 \mathrm{~mm}$ thick, which serves to be a reference plane for the micropipette to determine its relative height to the embryos for automatic robotic injection. It is based on the detection of the initial contact between the tip and the glass substrate. The contact detection is achieved by an automatic computer-vision-based method with an accuracy down to $0.2 \mu \mathrm{m}$ [17]. In this approach, the micropipette is lowered down by the microrobot to establish a physical contact with the glass substrate. Here, because of its smoothness, evenness, and transparency, the glass substrate rather than the device surface is used as the reference plane to eliminate two practical risks: i) micropipette tip breakage caused by micropipette buckling due to the rough surface of the device; and ii) scratch damage to the device surface due to the tip sliding.

The surface of the device was made by micromilling, and the through-holes were made by microdrilling. In microdrilling the through-holes, care must be taken to prevent overheating or melting of the material. Use of lubricant, $10000 \mathrm{rpm}$ rotation speed, and sharp drill bit avoids these issues.

The micromachined surface was polished using coarse and then fine sand paper, to obtain a good surface finish. The two parts were then washed several times using chloride to enhance its hydrophilicity, and then glued together after drying. The glass substrate was glued to the embryo sucking structure. The integral device (Fig. 6) was rinsed thoroughly using ethanol and then DI water for sterilization. The 3-D profile of the device surface was measured and the variation in flatness was used to vertically align the tip with embryos in injection process.

\section{Cell Patterning and Injection}

The device was connected to a venture vacuum pump (KNF filtration pump) which provided negative pressure. Initially, the pump was turned 'off'; and a number of embryos were transferred to the reservoir of the device. The pump was then turned 'on', and simultaneously the device was gently shaken back and forth by hand. In this process, more cell culture/media can be transferred to the reservoir to replenish the fluid flow. A couple of seconds later, the embryos were individually sucked into the throughhole array. And the non-trapped embryos flushed away. Fig. 7 shows this cell patterning procedure, which largely reduces the sample preparation time to 10-30 seconds, compared to 2-5 minutes for manual preparation.

The patterned embryos were injected sequentially by the automatic microrobotic cell injection system, which precisely recognizes the embryo structures and autonomously determines the deposition destinations.

Fluorescein-tagged morpholinos that target the gene no tail (ntl-MO, 5-GACTTGAGGCAGGCATATTTCCGAT-3, 300 $\mathrm{nM}$, Gene Tools) were injected into 210 patterned embryos, using this microrobotic cell injection system. The no tail gene product is required for tail formation in zebrafish [18]. Successful injection of ntl-MO inhibited translation of the ntl gene product, resulting in the tail-less phenotype, as shown in Fig. 8. The success rate, survival rate, and phenotypic rate were all close to $100 \%$, demonstrating that the pressurebased device is suitable for automating cell injection in any large or small scale application.

\section{DIELECTROPHORESIS-BASED CELL PATTERNING BIOCHIP}

The interaction of electric fields with biological cells (or particles, bacteria, viruses, DNA and proteins) by electrokinetics presents an electrically controllable method for manipulating living cells based on their unique dielectric properties [19]. Dielectrophoresis (DEP) is a phenomenon whereby suspended polarizable particles in a fluid can be made to move toward regions of high electric field strength or to be repelled from them. For a distinct and characterizable entity, dielectric properties will be unique and create a frequency dependent dielectrophoretic force, which attracts or repels the cells from the regions of higher or lower electric field gradients, respectively. Using negative DEP (-DEP), the cells can be repelled from the electrodes; conversely, the cells can be attracted to the electrode using positive DEP (+DEP). DEP is best suited to AC field. By changing the frequency of the $\mathrm{AC}$ field, both the direction and magnitude of the force can be modulated.

\section{A. DEP Biochip Design and Fabrication}

The DEP biochip is designed to have interdigitated electrodes, which apply AC field to attract or repel cells. Concentric to these electrodes are cavities, which are used to address 



Fig. 7. Sample preparation procedures under bright-field microscopy $(0.7 \times)$. (a) Random dispersed embryos on the surface. (b) Shaking of the device while the pressure applies. (c) Well-patterned embryos after extra embryos are flushed away.

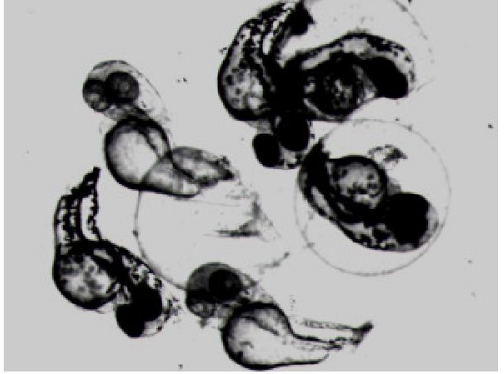

Fig. 8. Development of zebrafish embryos injected with fluorescen-tagged ntl-MO. $24 \mathrm{hr}$ after injection. Tail-less phenotype demonstrates that the negative pressure applied to the device does not affect the normal embryonic development.

and trap individual cells. Depending on the size of cell under study, the cavities can be designed with other dimensions and depth profile.

Electrodes microsystems and cavities are fabricated using two stage photolithography process as illustrated in Fig. 9. Firstly, a thin $20 \mathrm{~nm}$ adhesive film of nickel-chrome along with a $150 \mathrm{~nm}$ layer of gold are thermally evaporated onto a $100 \mathrm{~mm}$ wafer, insulated with either a $2 \mu \mathrm{m}$ thick siliconnitride layer, or comprised entirely of quartz (Silicon Quest Int'l). The electrodes are formed after gold, and then nickelchrome wet etching through a photolithography fabricated $1.5 \mu \mathrm{m}$ thick positive resist mask (AZ 1518, Clariant), which was developed for 15 seconds in AZ $300 \mathrm{MIF}$, Clariant developer, and UV exposed for 12 seconds using MA6 Karl Suss mask aligner with an unfiltered $350 \mathrm{~W}$ mercury lamp having an intensity at the centre of the stage of $6.7 \mathrm{~mW} / \mathrm{cm}^{2}$.

In the second stage of fabrication a mask defining an array of $10 \mu \mathrm{m}$ diameter single cell traps in the form of microcavities (microincubators) about $5 \mu \mathrm{m}$ deep are precisely aligned either between or above the interdigitated electrodes depending on the biochip design. For cavities above the electrodes a $1.5 \mu \mathrm{m}$ layer of photoresist was spun at 3000 rpm for 40 seconds and an isotropic profile created after a 28 seconds exposure and 60 seconds development process, respectively. Finally, the $100 \mathrm{~mm}$ wafer was hard-baked at $185^{\circ} \mathrm{C}$ for $1 \mathrm{hr}$ and diced into 24 individual biochips using diamond wafer dicer, for more details on biochip fabrication refer to [20].
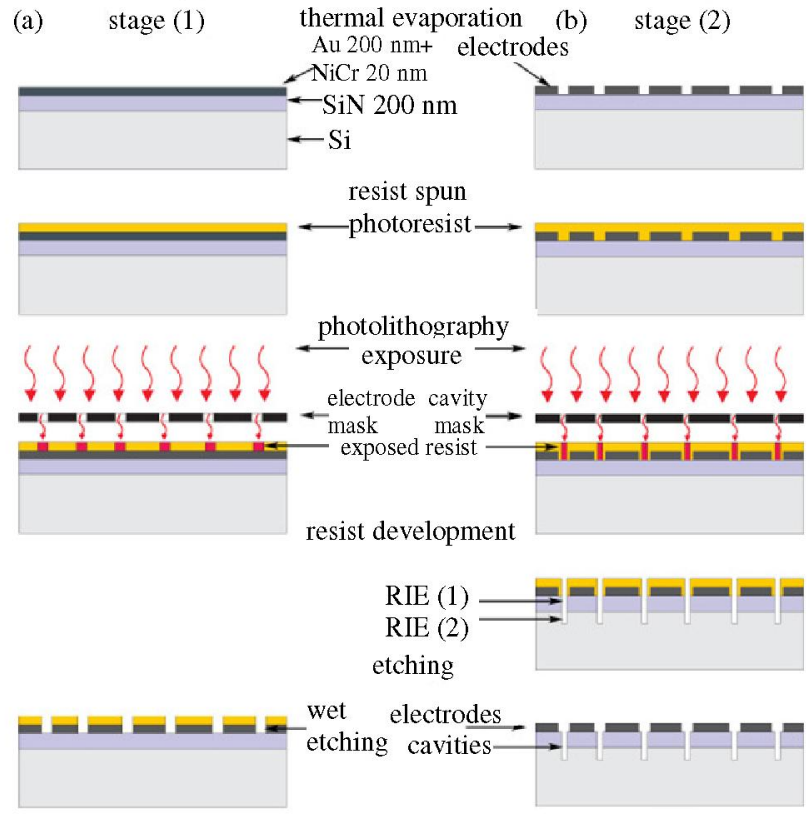

Fig. 9. DEP chip fabrication steps. (a) Formation of metal electrodes using photolithography and wet etching techniques. (b) Formation of cavities using dry etching process.

\section{B. Cell Trapping}

The Biochip consists of a series of interdigitated microelectrode structures fabricated for the manipulation of 5 to $10 \mu \mathrm{m}$ particles and biological cells. The dipole moments induced from the AC electric field combined with the field gradients generated from the non-uniform electrode structures, result in polarisation of the spherical particles. Polarisation causes particle movement either toward the high intense field regions (+DEP) or toward the low intense field regions (-DEP). Several microelectrode structures have been designed to test the unique DEP responses of different particles. As shown in Fig. 10, cavities placed between the sharp pointed electrodes are designed to trap particles that respond only to +DEP.

Good viability of cells trapped within cavities is demonstrated, and thus +DEP could be used as a positioning force to effectively trap living cells. The cavities may act as single-cell incubators, as well for other purposes (e.g., to address individual cells but also to facilitate Atomic Force Microscopy). Each cell will have a unique address and hence 


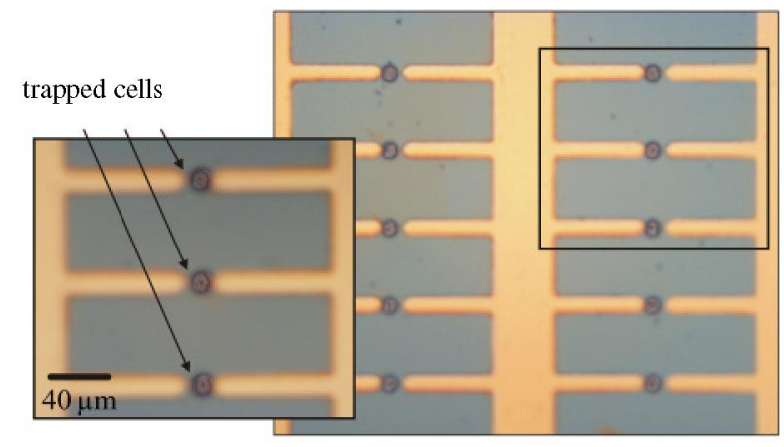

Fig. 10. Single cells trapped in cavities between Au electrodes.

may be individually analysed and monitored after injection. Because the address is known the analyis may be repeated at a regular time interval, adding significant potential value to many biological studies.

At optimal conditions defined by $\mathrm{AC}$ waveform characteristics(3-6 Vp-p at 500-800 kHz) and medium constituents, a suspension solution of biological cells is rapidly driven and attached to selective cavity locations using +DEP forces. Trypan blue testing demonstrated good viability of the cells trapped within these cavities.

Currently, the automatic injection system is being modified to inject the cells trapped on this DEP-based MEMS biochip.

\section{CONCLUSION}

Automatic microrobotic cell injection significantly reduces the inherent problems in manual injection and has many implications in enhancing molecular biology and drug development. In particular, it can eliminate the time-consuming and tedious manual patterning of suspended cells while also increasing throughput and accuracy. To cover the full size spectrum of suspended cells, two devices for patterning cells are presented in this paper. The pressure-based fluidic device provides a very low cost, easy-to-make, reusable method to pattern cells larger than $100 \mu \mathrm{m}$. Experimental results demonstrate that for this case the patterned cells can develop normally. Similarly, the DEP-based MEMS biochip provides a comparable solution to patterning cells smaller than 100 $\mu \mathrm{m}$. The high viability of trapped cells for this device proves its suitability for automatic cell injection. Overall, both devices can readily pattern cells into a regular grid up to 10 times faster than an operator and with greater precision, significantly improving throughput and quality.

\section{REFERENCES}

[1] M. P. Rols, "Electropermeabilization, a physical method for the delivery of therapeutic molecules into cells," Biochim. Biophys. Acta., vol. 1758 , no. 3, pp. $423-428,2006$.

[2] W. Walther and U. Stein, "Viral vectors for gene transfer: a review of their use in the treatment of human diseases," Drugs, vol. 60, no. 2, pp. 249-271, Aug. 2000.

[3] M. T. S. Lin, L. Pulkkinen, J. Uitto, and K. Yoon, "The gene gun: current applications in cutaneous gene therapy," Int. J. Dermatology, vol. 39, no. 3, pp. 161-170, Mar. 2000.
[4] J. Sundaram, B. R. Mellein, and S. Mitragotri, "An experimental and theoretical analysis of ultrasound-induced permeabilization of cell membranes," Biophys J., vol. 87, no. 2, pp. 1013-1033, 2004.

[5] S. Hashmi, P. Ling, G. Hashmi, M. Reed, R. Gaugler, and W. Trimmer, "Genetic transformation of nematodes using arrays of micromechanical piercing structures," BioTechniques, vol. 19, pp. 766-770, 1995.

[6] K. Chun, G. Hashiguchi, H. Toshiyoshi, H. Fujita, Y. Kikuchi, J. Ishikawa, Y. Murakami, and E. Tamiya, "An array of hollow micro-capillaries for the controlled injection of genetic materials into animal/plant cells," in Proc. IEEE International Conference on Micro Electro Mechanical Systems (MEMS'1999), 1999, pp. 406-411.

[7] W. H. Wang, X. Y. Liu, D. Gelinas, B. Ciruna, and Y. Sun, "A fully automated robotic system for microinjection of zebrafish embryos," PLoS ONE, vol. 2, no. 9, p. e862, Sept. 2007.

[8] M. Westerfield, The zebrafish book. Eugene, OR: University of Oregon Press, 1995.

[9] T. P. Hunt, H. Lee, and R. M. Westervelt, "Addressable micropost array for the dielectrophoretic manipulation of particle in fluid," App. Phy. Lett., vol. 85, no. 26, pp. 6421-6423, 2004.

[10] A. Ashkin, K. M. Dziedzic, and T. Yamane, "Optical trapping and manipulation of single cells using infrared laser beams," Nature, vol. 330, pp. 769-771, 1987.

[11] O. Muraoka, T. Shimizu, T. Yabe, H. Nojima, Y. K. Bae, H. Hashimoto, and M. Hibi, "Sizzled controls dorso-ventral polarity by repressing cleavage of the Chordin protein," Nature Cell Biology, vol. 8, pp. 329-340, Mar. 2006.

[12] D. H. Kim, C. N. Hwang, Y. Sun, S. H. Lee, B. Kim, and B. J. Nelson, "Mechanical analysis of chorion softening in prehatching stages of zebrafish embryos," IEEE Trans Nanobioscience, vol. 5, no, 2, pp. 89-94, June 2006.

[13] D. M. Langenau, M. D. Keefe1, N. Y. Storer, J. R. Guyon, J. L. Kutok, X. Le, W. Goessling, D. S. Neuberg, L. M. Kunkel, and L. I. Zon, "Effects of RAS on the genesis of embryonal rhabdomyosarcoma," Genes and Development, vol. 21, pp. 1275-1279, 2007.

[14] A. Nasevicius and S. C. Ekker, "Effective targeted gene 'knockdown' in zebrafish," Nature Genetics, vol. 26, pp. 216-220, 2000.

[15] J. Sasaki, S. Youoku, K. Tamamushi, and A. Ito, "Development of microchannel device for automated microinjection,", in Proc. SPIE, vol. 5718, 2005, pp. 186-193.

[16] X. Y. Liu, Y. Sun, W. H. Wang, and B. M. Lansdorp, "Vision-based cellular force measurement using an elastic microfabricated device," $J$. Micromechanics and Microengineering, vol. 17, no. 7, pp. 1281-1288, 2007.

[17] W. H. Wang, X. Y. Liu, and Y. Sun, "Contact detection in microrobotic manipulation," Int. J. Robot. Res., vol. 26, no. 8, pp. 821-828, 2007.

[18] S. Schulte-Merker, F. J. M. van Eeden, M. E. Halpern, C. B. Kimmel, and C. Nsslein-Volhard, "no tail ( $n t l)$ is the zebrafish homologue of the mouse T (Brachyury) gene," Development, vol. 120, pp. 1009-1015, 1994.

[19] M. P. Hughes, "Sorting cells and finding bugs with micro and nanoelectrokinetics," IEEE Medicine and Biology Magazine, vol. 22, no. 6, pp. 32-97, Nov/Dec 2003.

[20] J. Muys, M. M. Alkaisi, J. J. Evans, and J. Nagase, "Analysis of dielectrophoretically trapped biological cells by atomic force microscopy using an integrated biochip platform," Japanese Journal of Applied Physics, vol. 44, no. 7B, pp. 5717-5723, 2005. 